\title{
Voltammetric Determination of Bisphenol A Using a Carbon Paste Electrode Based on the Enhancement Effect of Cetyltrimethylammonium Bromide (CTAB)
}

\author{
Wensheng Huang \\ Department of Chemistry, Hubei Institute for Nationalities, Enshi 445000,P.R. China. E-mail: huang_wensh@hotmail.com \\ Received May 24, 2005
}

\begin{abstract}
The influence of cetyltrimethylammonium bromide (CTAB) on the electrochemical behavior of bisphenol A at the carbon paste electrode (CPE) was investigated. CTAB, with a hydrophobic $\mathrm{C}-\mathrm{H}$ chain, can adsorb at the CPE surface via hydrophobic interaction and then change the electrode/solution interface, and finally affects the electrochemical response of bisphenol A, confirming from the remarkable oxidation peak current enhancement. The electrode process of bisphenol A was examined, and then all the experimental parameters which affects the electrochemical response of bisphenol $\mathrm{A}$, such as $\mathrm{pH}$ value of the supporting electrolyte, accumulation potential and time, potential scan rate and the concentration of CTAB, were examined. Finally, a sensitive and simple voltammetric method was developed for the determination of bisphenol A. Under the optimum conditions, the oxidation peak current of bisphenol A varied linearly with its concentration over the range from $2.5 \times 10^{-8}$ to $1 \times 10^{-6} \mathrm{~mol} / \mathrm{L}$, and the detection limit was found to be $7.5 \times 10^{-9} \mathrm{~mol} / \mathrm{L}$. This method was successfully employed to determine bisphenol A in some waste plastic samples.
\end{abstract}

Key Words : Surfactant, Enhancement effect, Bisphenol A, Electrochemical detection, Carbon paste electrode

\section{Introduction}

Surfactant is a linear molecule with a hydrophilic (attracted to water) head and a hydrophobic (repelled by water) end. Due to its unique molecular structure, surfactant was extensively used in the fields of electrochemistry and electroanalytical chemistry for various purposes. ${ }^{1-6}$ Surfactants, containing hydrophobic and hydrophilic groups, can change the properties of the electrode/solution interface and subsequently influence the electrochemical processes of other substances. ${ }^{7}$ Adsorption of surfactant aggregates on the electrode surface might significantly facilitate the electron transfer, greatly enhance the peak current, change the redox potentials or charge transfer coefficients or diffusion coefficients, as well as alter the stability of electrogenerated intermediates or electrochemical products.

Bisphenol A, with the molecular structure as shown in Figure 1, is a major industrial chemical used primarily to make polycarbonate plastic and epoxy resins - both of which are used in countless applications that make our lives easier, healthier and safer. $^{8}$ Otherwise, there are several reports concerning the estrogenic activity of bisphenol A. ${ }^{9,10}$ Recently, in-vivo studies show that bisphenol A has the potential health effects on reproduction and generation. ${ }^{11-13}$ From its molecular structure, it is well-known that bisphenol A should possess electrochemical activity since it contains two electrochemical-active phenolic hydroxyl groups. So far, the electrochemical behaviors of bisphenol A at various electrodes such as carbon fiber electrode, ${ }^{14,15} \mathrm{Pt} / \mathrm{Ti}$ Electrode, ${ }^{16}$ have been examined, and then a variety of electrochemical methods for the detection of bisphenol A have also been reported. For example, high performance liquid chromatography with electrochemical detection ${ }^{17-19}$ and LCED with a chemically modified electrode ${ }^{20}$ were

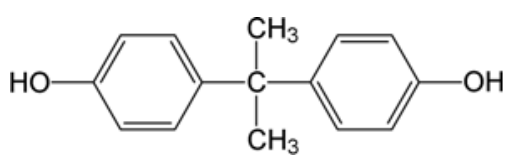

Figure 1. The molecular structure of bisphenol A.

successfully employed to detect bisphenol A. However, to the best of my knowledge, electrochemical determination of bisphenol A. using a carbon paste electrode in the presence of CTAB has not been reported.

CTAB, a widely-used surfactant, has been extensively employed to modify electrode. For instance, CTAB-covered carbon paste electrode was successfully used to immobilize DNA, ${ }^{21}$ to determine trace level of thyroxine. ${ }^{22}$ Otherwise, the influence of $\mathrm{CTAB}$ on the electrochemical properties of estrogens,${ }^{23,24}$ thiopurines, ${ }^{25}$ and vanadium $(\mathrm{v})^{26}$ has been reported.

The aim of the work is to establish a simple and sensitive electrochemical method for the determination of bisphenol $A$ based on the CTAB enhancement effect. In the presence of CTAB, the oxidation peak current of bisphenol A remarkably increases at the CPE suggesting significant improvement of determining sensitivity. The newly-proposed method possesses some obvious advantages including high sensitivity, extreme simplicity, rapid response and low cost.

\section{Experimental Section}

Reagents. The $1.0 \times 10^{-3} \mathrm{~mol} / \mathrm{L}$ stock solution of bisphenol A was prepared by dissolving bisphenol A (Shanghai Reagent Corporation, China) into ethanol. Cetyltrimethylammonium bromide (CTAB), graphite powder (spectral reagent) and paraffin oil were purchased from Shanghai 
Reagent Corporation, China. Other chemicals used were of analytical reagents. All the reagents were used as received without any further purification, and redistilled water was used.

Apparatus. All the electrochemical measurements were performed with a CHI 610A Electrochemical Workstation (CH instruments). A conventional three-electrode system, including carbon pasteworking electrode $(3 \mathrm{~mm}$ in diameter), a platinum wire counter electrode and a saturated calomel reference electrode (SCE), was employed.

Preparation of the carbon paste electrode. The carbon paste electrode was prepared by mixing $50 \mathrm{mg}$ graphite powder and $20 \mu \mathrm{L}$ paraffin oil in a small mortar, and this mixture was then homogenized. After that, the paste was pressed manually into the cavity of the electrode body, and the surface was smoothed against a clean paper. Unless otherwise stated, the paste was carefully removed prior to pressing a new portion into the electrode after every measurement.

Preparation of bisphenol A samples. The waste plastic samples were collected at a local disposal landfill site in Enshi. Plastic materials ( $c a .100 \mathrm{~g}$ ) were cut into small pieces and transferred into a $500 \mathrm{~mL}$ glassy beaker containing $300 \mathrm{~mL}$ of water and sealed with aluminum foil. The samples were left in dark at room temperature for two weeks. Bisphenol A was determined in the leachate water samples by the standard addition method, i.e. by adding a very small volume of relatively concentrated bisphenol A standard solution, so that the dilution effect could be neglected in calculations.

Experimental procedure. Prior to each measurement, the carbon paste electrode was activated in an electrochemical cell containing $0.1 \mathrm{~mol} / \mathrm{L}$ phosphate buffer $(\mathrm{pH} 6.5,10 \mathrm{~mL})$ by cyclic voltammetric sweeps between 0.00 and $1.20 \mathrm{~V}$ until the cyclic voltammograms were stable. After that, a certain concentration of bisphenol $A$ and $6.0 \times 10^{-5} \mathrm{~mol} / \mathrm{L}$ $\mathrm{CTAB}$ were added into, then the voltammograms were recorded for bisphenol $\mathrm{A}$.

\section{Results and Discussion}

The voltammetric behavior of bisphenol $A$ at a carbon paste electrode. The electrochemical behavior of bisphenol A in $\mathrm{pH} 6.5$ phosphate buffer was examined using cyclic voltammetry $(\mathrm{CV})$, and the cyclic voltammograms shown in Figure 2. In the absence of CTAB, $1 \times 10^{-6} \mathrm{~mol} / \mathrm{L}$ bisphenol A yields an oxidation peak at the carbon paste electrode (curve a). The peak potential is at $0.92 \mathrm{~V}$ and the peak height is very low (just about $0.15 \mu \mathrm{A}$ ). However, the electrochemical response of bisphenol A shows great difference in the presence of $6.0 \times 10^{-5} \mathrm{~mol} / \mathrm{L}$ CTAB under the identical conditions (curve b). The main differences are as follows: (i) the oxidation peak current increases greatly (from $0.15 \mu \mathrm{A}$ to $3.42 \mu \mathrm{A}$ ); (ii) the peak potential shifts negatively to 0.87 $\mathrm{V}$. In curves (a) and (b), no corresponding reduction peak is observed on the reverse sweep, indicating that the electrode reaction of bisphenol $\mathrm{A}$ is an irreversible process.

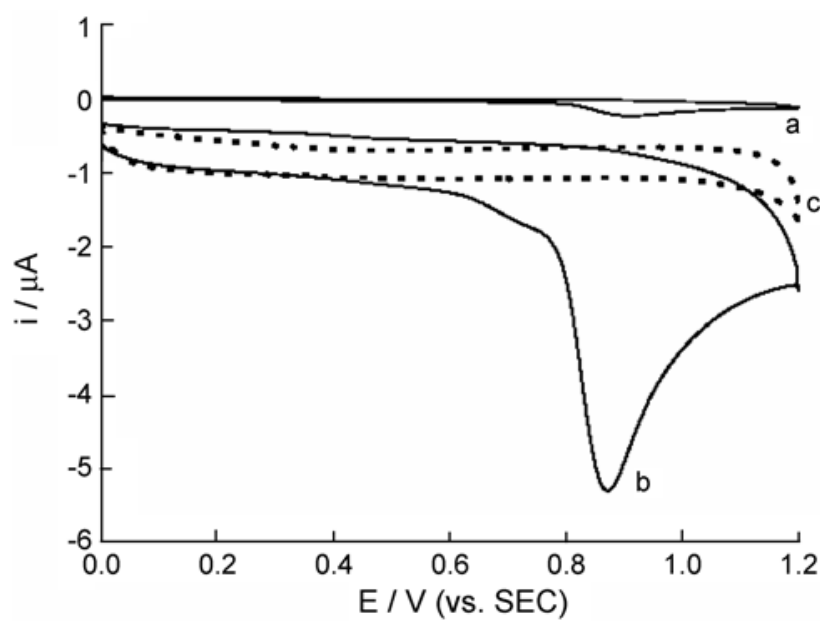

Figure 2. The cyclic voltammetry of $1.0 \times 10^{-6} \mathrm{~mol} / \mathrm{L}$ bisphenol A at a caron paste electrode in the absence of CTAB (curve a) and in the presence of $6.0 \times 10^{-5} \mathrm{~mol} / \mathrm{L}$ CTAB (curve b). Curve c: cyclic voltammetry of a carbon paste electrode in the presence of $6.0 \times$ $10^{-5} \mathrm{~mol} / \mathrm{L}$ CTAB but without bisphenol A. Scan rate: $100 \mathrm{mV} / \mathrm{s}$.

Otherwise, the cyclic voltammogram of a carbon paste electrode in $\mathrm{pH} 6.5$ phosphate buffer and in the presence of $6.0 \times 10^{-5} \mathrm{~mol} / \mathrm{L} \mathrm{CTAB}$ was recorded to compare (curve c). No redox peak is observed, suggesting that the oxidation peak at $0.87 \mathrm{~V}$ in curve (b) is undoubtedly assigned to bisphenol A. The comparison of curves (a) and (b) tells that $\mathrm{CTAB}$ can influence the electrochemical process of bisphenol A and facilitate its electron transfer.

Influences of $\mathbf{p H}$. The effect of $\mathrm{pH}$ value on the oxidation peak potential $\left(\mathrm{E}_{\mathrm{pa}}\right)$ and peak currernt $\left(\mathrm{i}_{\mathrm{pa}}\right)$ of bisphenol $\mathrm{A}$ in the presence of CTAB was investigated using linear sweep voltammetry (LSV) from 0.20 to $1.20 \mathrm{~V}$. As illustrated in Figure 3 , during $\mathrm{pH}$ value improving from 5.0 to $8.0, \mathrm{i}_{\mathrm{pa}}$ gradually increases as $\mathrm{pH}$ value increasing from 5.0 to 6.5 , and then shows decline as further improving $\mathrm{pH}$ value.

As far as peak potential is concerned, it gradually shifts towards negative potential with increasing $\mathrm{pH}$ value from

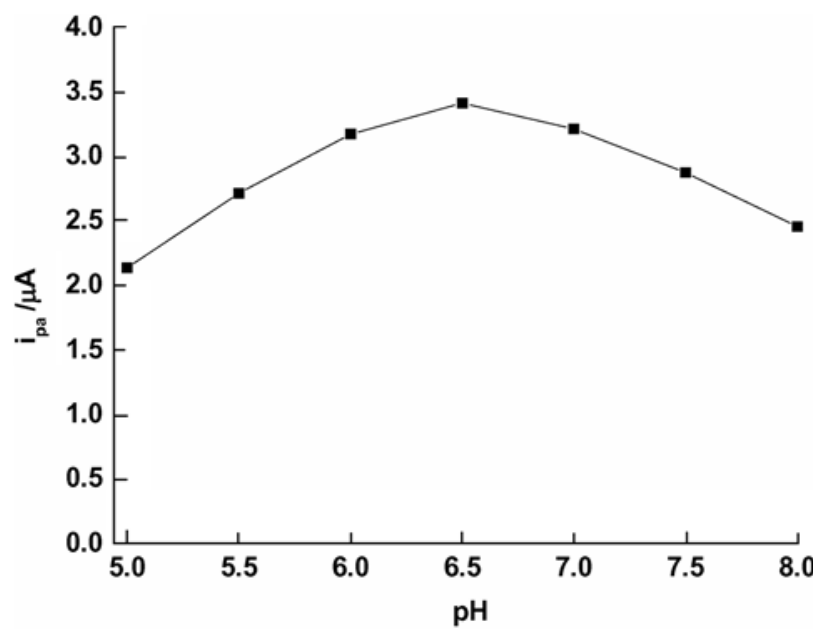

Figure 3. Plots of the oxidation peak current of $1.0 \times 10^{-6} \mathrm{~mol} / \mathrm{L}$ bisphenol A versus the $\mathrm{pH}$ value. CTAB concentration: $6.0 \times 10^{-5}$ $\mathrm{mol} / \mathrm{L}$; scan rate: $100 \mathrm{mV} / \mathrm{s}$. 


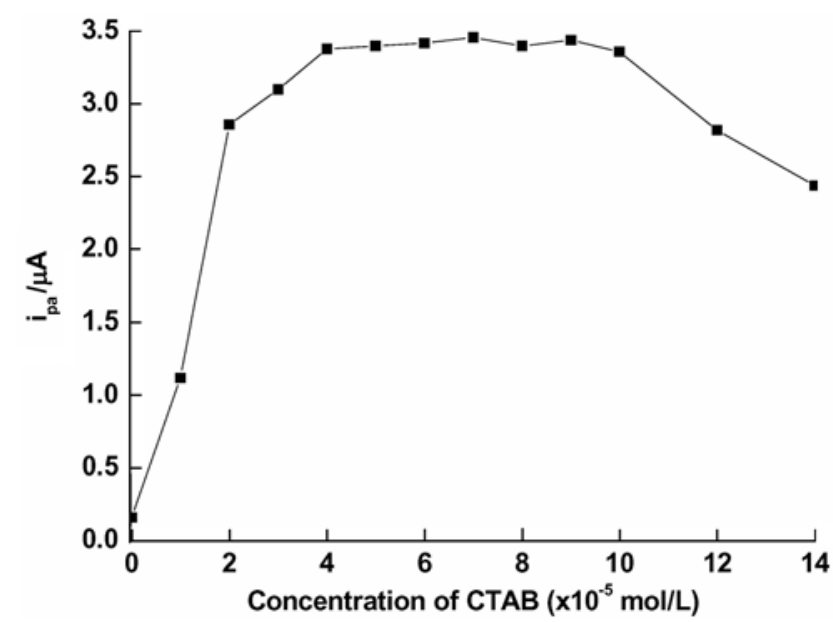

Figure 4. The influence of $\mathrm{CTAB}$ concentration on the oxidation peak current of $1.0 \times 10^{-6} \mathrm{~mol} / \mathrm{L}$ bisphenol A.

5.0 to 8.0. Further studies indicate that the slopes of $\mathrm{E}_{\mathrm{pa}} / \mathrm{pH}$ in the presence and absence of CTAB are about $-58.5 \mathrm{mV} /$ $\mathrm{pH}$ and $-58.1 \mathrm{mV} / \mathrm{pH}$, respectively. The slopes reveals that equal number of protons and electrons are involved in the oxidation of bisphenol A. Moreover, the slopes of $\mathrm{E}_{\mathrm{pa}} / \mathrm{pH}$ in the presence and absence of CTAB are very close, suggesting that $\mathrm{CTAB}$ does not alter the mechanism of electrochemical oxidation of bisphenol $\mathrm{A}$ at all. In this work, a $\mathrm{pH}$ of 6.5 phosphate buffer was selected as supporting electrolyte.

The concentration of CTAB. Figure 2 clearly tells that CTAB exhibits remarkable enhancement effect on the oxidation peak current of bisphenol A. However, the oxidation peak current of bisphenol A is closely related to the concentration of CTAB. Figure 4 depicts the relationship between the oxidation peak current of bisphenol $\mathrm{A}$ and the concentration of CTAB. The oxidation peak current increases greatly as CTAB concentration improving from 0 to $4 \times 10^{-5} \mathrm{~mol} / \mathrm{L}$, and then increases slightly over the range from $4 \times 10^{-5}$ to $1 \times 10^{-4} \mathrm{~mol} / \mathrm{L}$, and finally decreases when CTAB concentration is higher than $1 \times 10^{-4} \mathrm{~mol} / \mathrm{L}$. Meanwhile, the background current gradually increases while improving the $\mathrm{CTAB}$ concentration. It is known that CTAB bulk concentration determines its existing form in aqueous solution as well as its arrangement at the carbon paste electrode/solution interface, which certainly influence the electrochemical response of bisphenol A. In this work, the concentration of CTAB was chosen to be $6 \times 10^{-5} \mathrm{~mol} / \mathrm{L}$ for a higher peak current and a lower background current.

Electrode process of bisphenol $\mathbf{A}$. The effect of varying the potential scan rate on the oxidation peak current of bisphenol A was examined by LSV. The oxidation peak currents in the presence and absence of CTAB increased linearly with scan rate over the range from $25 \mathrm{mV} / \mathrm{s}$ to 300 $\mathrm{mV} / \mathrm{s}$, suggesting that the oxidation of bisphenol $\mathrm{A}$ at the carbon paste electrode is adsorption-controlled.

As for an adsorption-controlled and irreversibly electrode process, according to Laviron, the oxidation peak potential $\left(E_{\mathrm{pa}}\right)$ is described by the following equation: $:^{27}$
$E_{\mathrm{pa}}=E^{0^{\prime}}+(2.303 \mathrm{R} T / n \mathrm{~F}) \lg \left(\mathrm{R} T k^{0} / n \mathrm{~F}\right)+(2.303 \mathrm{R} T / n \mathrm{~F}) \lg v$

Where $\alpha$ is the transfer coefficient, $k^{0}$ the standard rate constant of the reaction, $n$ the electron transfer number, $v$ the scan rate, and $E^{0}$ the formal redox potential. Other symbols have their usual meaning. The relationship between $E_{\mathrm{pa}}$ and $v$ in the presence of CTAB was examined using LSV and $E_{\mathrm{pa}}$ depends linearly on the logarithm of $v$ according to equation: $E_{\mathrm{pa}}(\mathrm{V})=0.9316+0.0621 \log v, \mathrm{R}=0.996$. The value of $\alpha n$, calculated from the slope of the plot of 0.0621 , is 0.961 . Generally, $\alpha$ is assumed to be 0.5 in a totally irreversible electrode process. Thus, two electrons are involved in electro-oxidation of bispehnol A. The $\mathrm{pH}$ effect on $E_{\mathrm{pa}}$ indicates the number of electrons and protons involved in the oxidation process of bisphenol $\mathrm{A}$ is equal. Therefore, the electro-oxidation of bisphenol $\mathrm{A}$ in the presence of $\mathrm{CTAB}$ is a two-electron and two-proton process.

Otherwise, the oxidation peak potential $\left(E_{\mathrm{pa}}\right)$ of bisphenol $A$ in the absence of CTAB was also measured under different potential scan rate using linear sweep voltammetry, and the similar result was obtained, i.e. the electrode process of bisphenol $\mathrm{A}$ in the absence of CTAB is also a twoelectron and two-proton process

Effects of the accumulation potential and time. The influence of accumulation potential on the oxidation peak current of bisphenol $\mathrm{A}$ in the presence and absence of CTAB was studied. The peak current almost remained stable when the accumulation potential shifted from $-0.50 \mathrm{~V}$ to $0.50 \mathrm{~V}$, revealing that the accumulation potential has no obvious effect on the oxidation peak current. Thus, the accumulation of bisphenol A was performed under the open-circuit condition.

Unlike accumulation potential, accumulation time heavily influences the oxidation peak current of bisphenol A. The influences of the accumulation time on the peak current in the presence and absence of CTAB have been examined by linear sweep voltammetry, and the results shown in Figure 5.

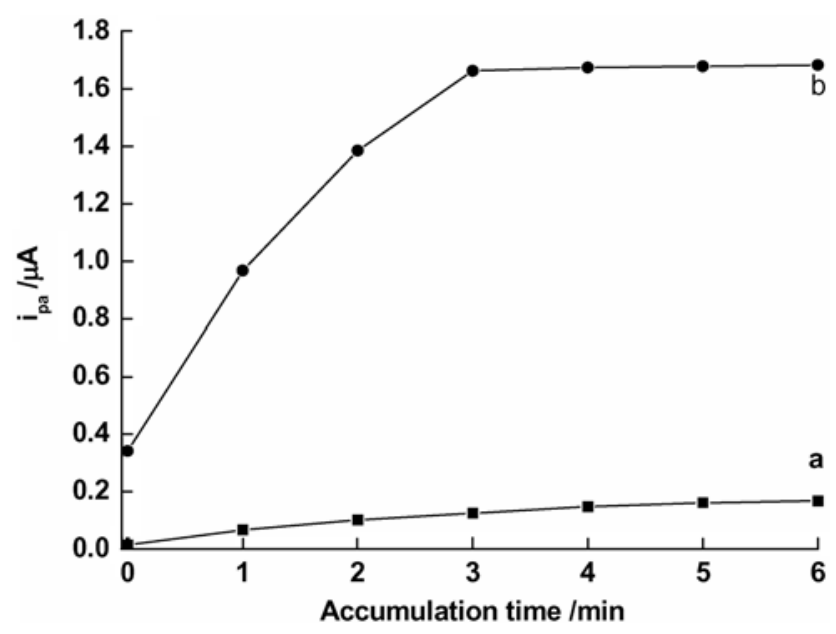

Figure 5. Effects of accumulation time on the the oxidation peak current of $1.0 \times 10^{-7} \mathrm{~mol} / \mathrm{L}$ bisphenol $\mathrm{A}$ in the absence of CTAB (curve a) and in the presence of $6.0 \times 10^{-5} \mathrm{~mol} / \mathrm{L} \mathrm{CTAB}$ (curve b). 


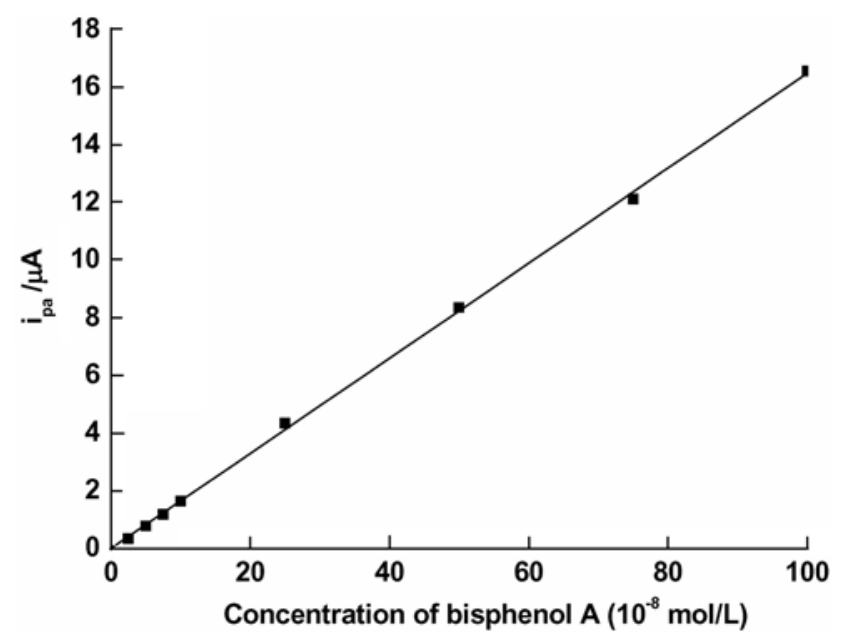

Figure 6. The calibration curve of bisphenol A from $2.5 \times 10^{-8}$ to $1.0 \times 10^{-6} \mathrm{~mol} / \mathrm{L}$.

The peak current increased linearly with the accumulation time over the range of 0.1-3 min. However, with further increasing the accumulation time, the plot became curved. The curvature presumably indicates that a limiting value of the amount of bisphenol A on the surface of the electrode had been achieved under the adopted conditions. A further accumulation time increase did not improve the amount of bisphenol $\mathrm{A}$ at the electrode surface owing to surface saturation. So the peak current almost remained constant. The sensitivity for lower concentrations was improved by increasing the accumulation time, but the linear range was then diminished. Otherwise, it is very clear that the peak current increases greatly in the presence of CTAB (curve b) compared with that in the absence of CTAB (curve a).

Response to bisphenol A. Differential pulse voltammetry (DPV) is an effective and common-used electroanalytical technique when the content of analyte is very low since it possesses high sensitivity. The relationship between the oxidation peak current of bisphenol A and its concentration was investigated using DPV. The best parameters on the carbon paste electrode are accumulation time $=3 \mathrm{~min}$, pulse amplitude $=50 \mathrm{mV}$, scan rate $=20 \mathrm{mV} / \mathrm{s}$, pulse width $=50$ $\mathrm{ms}$, concentration of CTAB $=6 \times 10^{-5} \mathrm{~mol} / \mathrm{L}$. Figure 6 shows the calibration curve of bisphenol $\mathrm{A}$ under the optimum conditions. It is found that the oxidation peak current $\left(\mathrm{i}_{\mathrm{pa}}\right)$ varies linearly with the concentration over the range from $2.5 \times 10^{-8}-1 \times 10^{-6} \mathrm{~mol} / \mathrm{L}$, obeying the following equation: $\mathrm{i}_{\mathrm{pa}}=0.018+0.165 \times 108 \mathrm{C}(\mathrm{r}=0.9989, \mathrm{C}$ in
Table 1. Interferences of other speicies on $5.0 \times 10^{-7} \mathrm{~mol} / \mathrm{L}$ bisphenol A

\begin{tabular}{ccc}
\hline Interferents & Concentration $(\mathrm{mol} / \mathrm{L})$ & $\mathrm{i}_{\mathrm{pa}}$ change $(\%)$ \\
\hline $\mathrm{Fe}^{2+}$ & $1.0 \times 10^{-5}$ & -2.6 \\
$\mathrm{~Pb}^{2+}$ & $1.0 \times 10^{-5}$ & 2.5 \\
$\mathrm{Zn}^{2+}$ & $1.0 \times 10^{-5}$ & -1.7 \\
$\mathrm{Cu}^{2+}$ & $1.0 \times 10^{-5}$ & -2.2 \\
$\mathrm{Al}^{3+}$ & $1.0 \times 10^{-5}$ & 2.8 \\
$\mathrm{Hg}^{2+}$ & $1.0 \times 10^{-5}$ & 2.8 \\
$\mathrm{CO}_{3}^{2-}$ & $1.0 \times 10^{-5}$ & -2.1 \\
$\mathrm{Cl}^{-}$ & $1.0 \times 10^{-5}$ & -1.3 \\
$\mathrm{NO}_{3}^{-}$ & $1.0 \times 10^{-5}$ & 1.5 \\
2,4-Dinitrophenol & $5.0 \times 10^{-6}$ & 5.4 \\
2(4)-Nitrophenol & $5.0 \times 10^{-6}$ & -4.6 \\
Phenol & $1.0 \times 10^{-6}$ & 4.1 \\
2-Aminophenol & $5.0 \times 10^{-6}$ & 2.3 \\
Ethanol & $1.0 \times 10^{-5}$ & -2.4 \\
\hline
\end{tabular}

$\mathrm{mol} / \mathrm{L}, \mathrm{i}_{\mathrm{pa}}$ in $\left.\mu \mathrm{A}\right)$. Under the optimized conditions, the detection limit of the method is as low as $7.5 \times 10^{-9} \mathrm{~mol} / \mathrm{L}$. The relative standard deviation (RSD) of $5.6 \%$ for $5 \times 10^{-7}$ $\mathrm{mol} / \mathrm{L}$ bisphenol A $(\mathrm{n}=7)$ shows good reproducibility.

Interferences and Sample Analysis. The effect of various possible interferents was examined on the determination of bisphenol A. The results are listed in Table 1. It is found that lots of species do not interfere with the determination of bisphenol A up to a 2-to-20-fold excess (signal change $<5 \%$ ).

Bisphenol A was determined in four types of plastic waste samples (Table 2). The results were in good agreement with the reference values obtained by GC/MS. The recoveries obtained using our procedure were also good enough for practical use, suggesting that this newly-proposed method has great potential in practical sample analysis. From the Table 2, we can see that concentration of bisphenol A that leached from the pure water container (sample $\mathrm{A}$ ) and the compact disk (sample B), both polycarbonate products, is relatively low. On other hand, however, bisphenol A that leached from the poly(vinyl chloride) product (sample C) and synthetic leather (sample D) was high.

\section{Conclusion}

Well below the critical micelle concentration (CMC), surfactants may adsorb individually and have little interaction with each another. ${ }^{28}$ For ionic surfactants adsorption

Table 2. Determination of bisphenol A in plastic waste samples

\begin{tabular}{ccccc}
\hline No. & Samples & $\begin{array}{c}\text { Detected by GC/MS } \\
\left(\mu \mathrm{g} \mathrm{dm}^{-3}\right)\end{array}$ & $\begin{array}{c}\text { Detected by this method } \\
\left(\mu \mathrm{g} \mathrm{dm}^{-3}\right)\end{array}$ & $\begin{array}{c}\text { Recovery of this method } \\
(\%)\end{array}$ \\
\hline A & Pure water container, (Polycarbonate) & 13.4 & 12.6 & 102.6 \\
B & Compact disk (Polycarbonate) & 18.3 & 18.9 & 99.4 \\
C & Electrical cord, [Poly(vinyl chloride)] & 495 & 493 & 101.8 \\
D & Synthetic leather & 2116 & 2117 & 101.4 \\
\hline
\end{tabular}



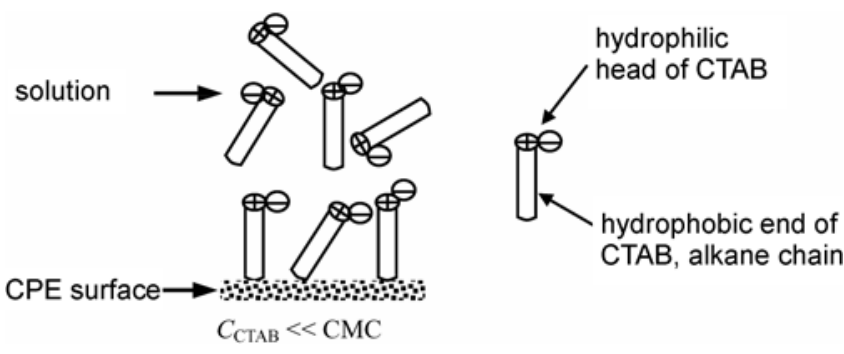

Figure 7. Schematic representation of low concentration of CTAB (well about $\mathrm{CMC}$ ) adsorbs at $\mathrm{CPE} /$ aqueous solution interface. CTAB molecules adsorb with their alkane chains extended on the substrate plane.

on the hydrophobic surface, there is a general agreement that the lower-density adsorbed layer (below the CMC) is orientated with surfactants tails parallel to the substrate plane, driven by a strong hydrophobic interaction between the alkane tails and the substrate, which have been verified by atomic force microscope (AFM). ${ }^{29}$ In this work, the concentration of $\mathrm{CTAB}$ is as low as $6.0 \times 10^{-5} \mathrm{~mol} / \mathrm{L}$ (well below $\left.\mathrm{CMC}=9.0 \times 10^{-4} \mathrm{~mol} / \mathrm{L}\right)$. According to references [21], [28] and [29], the orientation of CTAB on the CPE/ solution interface in this work can be illustrates as Figure 7. CTAB molecules adsorb on the CPE surface individually via hydrophobic interaction, then alters the structure of CPE/ solution interface, and finally affects the electrochemical activity of bisphenol A. Therefore, the electron transfer between bisphenol $\mathrm{A}$ and the carbon paste electrode becomes easily since the oxidation peak current increased remarkably accompanied by a lowering of the oxidation overpotential.

Acknowledgements. This work is financially supported by the Natural Science Foundation of Hubei Provincial Department of Education (D200529004).

\section{References}

1. Hu, S. S.; He, Q.; Zhao, Z. F. Anal. Chim. Acta 1991, 258, 103.

2. Rusling, J. F.; Nassar, A.-E. F. J. Am. Chem. Soc. 1993, 115, 11891.
3. Gao, J. X.; Rusling, J. F. J. Electroanal. Chem. 1998, 449, 1.

4. Yang, J.; Hu, N. F.; Rusling, J. F. J. Electroanal. Chem. 1999, 463, 53.

5. Wen, X. L.; Jia, Y. H.; Liu, Z. L. Talanta 1999, 50, 1027.

6. Zhang, S. H.; Wu, K. B. Bull. Korean Chem. Soc. 2004, 25, 1321.

7. Plavsic, M.; Krznaric, D.; Cosovic, B. Electroanalysis 1994, 6, 469.

8. Summerfield, W.; Goodson, A.; Cooper, I. Food Addit. Contam. 1998, 15,818

9. Krishnan, A.; Stathis, P.; Permuth, S.; Tokes, L.; Feldman, D. Endocrinology 1993, 132, 2279.

10. Brotons, J.; Olea-Serrano, M.; Villalobos, M.; Pedraza, V.; Olea, N. Environ. Health Perspect. 1995, 103, 608.

11. Howdeshell, K. L.; Hotchkiss, A. K.; Thayer, K. A.; Vandenbergh, J. G.; Vom Saal, F. S. Nature 1999, 401, 763.

12. Nagel, S. C.; Vom Saal, F. S.; Thayer, K. A.; Dhar, M. G.; Boechler, M.; Welshons, W. V. Environ. Health Perspect. 1997, 105,70 .

13. Steinmetz, R.; Mitchner, N. A.; Grant, A.; Allen, D. L.; Bigsby, R. M.; Ben-Jonathan, N. Endocrinology 1998, 139, 2741.

14. Kuramitz, H.; Nakata, Y.; Kawasaki, M.; Tanaka, S. Chemosphere 2001, 45, 37.

15. Kuramitz, H.; Matsushita, M.; Tanaka, S. Water Research 2004, $38,2330$.

16. Tanaka, S.; Nakata, Y.; Kimura, T.; Yustiawati, T.; Kawasaki, M.; Kuramitz, H. J. Appl. Electrochem. 2002, 32, 197.

17. Inoue, K.; Kato, K.; Yoshimura, Y.; Makino, T.; Nakazawa, H. J. Chromatogr. B: Biomed. Sci. Appl. 2001, 749, 17.

18. Sajiki, J. J. Chromatogr. B: Biomed. Sci. Appl. 2001, 755, 9.

19. Ishiyama, T.; Shimadu, T.; Nudeshima, C.; Hoshi, J.; Sasaki, Y. Bunseki kagaku (Japan Analyst) 2004, 53, 411.

20. Antuono, A. D.; Campo, D. V.; Balbo, A. L.; Sobral, S.; Rezzano, I. J. Agricultural and Food Chem. 2001, 49, 1098.

21. Hu, C. G.; Hu, S. S. Electrochimica Acta 2004, 49, 405.

22. Hu, C. G.; Dang, X. P.; Hu, S. S. J. Electroanal. Chem. 2004, 572, 161.

23. Hu, S. S.; Wu, K. B.; Yi, H. C.; Cui, D. F. Anal. Chim. Acta 2002 , 464, 209.

24. He, Q.; Yuan, S.; Chen, C.; Hu, S. S. Materials Science and Engineering C 2003, 23, 621 .

25. Zeng, B.; Purdy, W. C. Electroanalysis 1999, 11, 879.

26. Stadlober, M.; Kalcher, K.; Raber, C. Electroanalysis 1997, 9, 225.

27. Laviron, E. J. Electroanal. Chem. 1974, 52, 355.

28. Rusling, J. F. Colloids Surfaces A: Physicochem. Eng. Aspects 1997, 123/124, 81.

29. Manne, S.; Cleveland, J. P.; Gaub, H. E.; Stucky, G. D.; Hansma, P. K. Langmuir 1994, 10, 4409. 